\title{
Insulin Resistance and Muscle Weakness Are Synergistic Risk Factors for Silent Lacunar Infarcts: the Bunkyo Health Study: a Cross Sectional Study
}

Yuki someya

Juntendo University

Yoshifumi Tamura ( $\square$ ys-tamur@juntendo.ac.jp )

Juntendo University: Juntendo Daigaku https://orcid.org/0000-0002-1685-7821

Hideyoshi Kaga

Juntendo University

Daisuke Sugimoto

Juntendo University

Satoshi kadowaki

Juntendo University

Ruriko Suzuki

Juntendo University

Shigeki Aoki

Juntendo University

Nobutaka Hattori

Juntendo University

Yumiko Motoi

Juntendo University

Kazunori Shimada

Juntendo University

Hiroyuki Daida

Juntendo University

Muneaki Ishijima

Juntendo University

Kazuo Kaneko

Juntendo University

Shuko Nojiri

Juntendo University

Ryuzo Kawamori

Juntendo University

Hirotaka Watada 
Juntendo University

\section{Original investigation}

Keywords: Insulin sensitivity, Muscle strength, Silent lacunar infarcts, combined effect, Community-based study

Posted Date: March 16th, 2021

DOl: https://doi.org/10.21203/rs.3.rs-297654/v1

License: (c) (1) This work is licensed under a Creative Commons Attribution 4.0 International License. Read Full License 


\section{Abstract}

Background: Previous studies showed that insulin resistance and reduced muscle strength are risk factors for silent lacunar infarcts, but it is unclear whether they are still independent risk factors when adjusted for each other. In addition, the effect of their combination on silent lacunar infarcts is completely unknown.

Methods: We evaluated silent lacunar infarcts, insulin sensitivity, and knee extensor muscle strength by magnetic resonance imaging, PREDIM (an index for insulin sensitivity), and dynamometer, respectively, in 1531 elderly people aged 65-84 years living in an urban area of Tokyo.

Results: Among the study subjects, 251 (16.4\%) had silent lacunar infarcts. Impaired insulin sensitivity (High; 1.00 [reference], Medium; 1.53 [95\% confidence interval (Cl): 0.94-2.48], Low; 1.86 [95\%Cl: 1.023.39], $\mathrm{p}$ for trend 0.047) and reduced muscle strength (High; 1.00 [reference], Medium; 1.40 [95\% Cl: 0.982.02], Low; 1.49 [95\% Cl: 1.04-2.15], p for trend 0.037) were independently associated with increased risk for silent lacunar infarcts in the fully adjusted model. In terms of combined, subjects classified as having the lowest insulin sensitivity and lowest strength were 4.33 times (95\% Cl: $1.64-11.45)$ more likely to have a silent lacunar infarct than those classified as having the highest insulin sensitivity and highest strength.

Conclusions: Impaired insulin sensitivity and reduced muscle strength were independently associated with higher risk of silent lacunar infarcts in elderly subjects, and their combination synergistically increased this risk.

\section{Background}

Lacunar infarcts are often incidentally found on brain magnetic resonance imaging (MRI) scans in asymptomatic elderly people, and in such cases they are defined as silent lacunar infarcts. Some epidemical studies revealed that over a quarter of elderly Japanese people have silent brain infarcts (1$4)$, and it has been shown that they are a risk factor for fatal and non-fatal stroke $(5,6)$, dementia (7) and frailty (8). In particular, subjects with silent lacunar infarcts in a Japanese cohort had a 10.48-fold increased risk of incident stroke (6). Therefore, prevention of silent lacunar infarcts might be an important strategy to prevent future stroke, dementia, and frailty in elderly subjects, especially subjects with diabetes because of their increased risk of stroke, dementia, and frailty (9). However, the overall etiology of silent brain infarcts remains unclear, though aging and hypertension are recognized as reliable risk factors $(1,10)$.

Several studies have shown that metabolic syndrome is strongly associated with silent brain infarcts ( 1 , 11 ), increasing their risk by 2.4 -fold in one report (11). Because insulin resistance is the fundamental condition underlying the pathogenesis of metabolic syndrome $(12,13)$, it is also a risk factor for silent lacunar infarcts. In fact, Lee et al. demonstrated in a cross-sectional study that subjects with insulin resistance, defined as over 2.56 times the cutoff of the homeostasis model assessment of insulin resistance (HOMA-IR), had a 1.69-fold higher risk of silent lacunar infarcts than subjects without insulin 
resistance (14). No other research has investigated the direct relationship between insulin resistance and silent lacunar infarcts.

Several studies, however, have demonstrated that reduced muscle strength is a risk factor for silent lacunar infarcts. Indeed, it was reported that low muscle strength at age 18 years was a risk factor for future ischemic stroke (15). Lower handgrip strength in middle age also increased the risk of cerebral infarction (16). Very recently, we revealed that reduced muscle strength was associated with a higher risk for silent lacunar infarcts (17). Given that skeletal muscle is the main organ that determines insulin sensitivity (13), impaired muscle functions such as insulin resistance and reduced muscle strength seem to be risk factors for silent lacunar infarcts. However, it is unclear whether they act independently of one another, since insulin sensitivity is weakly but positively associated with muscle strength $(18,19)$. In addition, even though muscle strength and insulin sensitivity are independent risk factors, it remains unclear how their combinations predict the incidence of silent lacunar infarcts.

Given the above background, the present study investigated whether reduced muscle strength and insulin sensitivity are independent risk factors for silent lacunar infarcts, using the data of 1531 elderly subjects aged 65-84 years who participated in the Bunkyo Health Study. In addition, we evaluated the combined effect of insulin sensitivity and muscle strength on silent lacunar infarcts.

\section{Materials And Methods}

\section{Study design and participants}

The Bunkyo Health Study is a prospective cohort study designed to clarify how muscle mass, muscle strength, and insulin sensitivity are associated with multiple diseases that necessitate long-term care (20). In this study, we recruited elderly subjects aged 65-84 years living in Bunkyo-ku, an urban area in Tokyo, Japan. All subjects participated in examinations over two visits to the Sportology Center from October 15, 2015, to October 1, 2018. Briefly, we evaluated cognitive function by questionnaires, physical fitness by dynamometer and physical performance tests, brain lesions by MRI, body composition and bone mineral density by dual-energy X-ray absorptiometry (DXA), arteriosclerosis by the cardio-ankle vascular index (CAVI), and abdominal fat distribution by MRI. We also carried out a 75-g oral glucose tolerance test (OGTT). This study protocol was approved by the ethics committee of Juntendo University in November 2015 (Nos. 2015078, 2016138, 2016131, 2017121, and 2019085). This study is carried out in accordance with the principles outlined in the Declaration of Helsinki. All participants gave written informed consent at the orientation meeting. Participants were told that they had the right to withdraw from the trial at any time.

This study enrolled 1612 subjects who had undergone OGTT, muscle strength testing, and brain MRI at the beginning of the Bunkyo Health Study. Missing data were relatively infrequent for OGTT $(0.3 \%)$, muscle strength $(0.3 \%)$, and MRI $(0.4 \%)$. Among the 1612 subjects, 81 with a previous stroke event were excluded; these subjects either had a previous confirmed clinical stroke $(n=52)$ or newly found non- 
lacunar brain infarcts by MRI in the Bunkyo Health Study $(n=29)$. Therefore, 1531 subjects were analyzed.

\section{Evaluation of insulin sensitivity}

Insulin sensitivity was estimated by PREDIM, a recently established index for insulin sensitivity (21). PREDIM was calculated from the Oral Glucose Insulin Sensitivity (OGIS) index (22) and other parameters, including body mass index (BMI), 2-h glucose during OGTT, and fasting insulin, to achieve good correlation with insulin sensitivity ( $M$ value) measured by hyperinsulinemic euglycemic clamp.

The OGIS is calculated using the following equation:

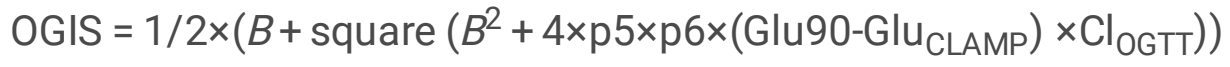

$B=\left(\mathrm{p} 5 \times\left(\mathrm{Glu} 90-\mathrm{Glu}_{\mathrm{CLAMP}}\right)+1\right) \times \mathrm{Cl}_{\mathrm{OGTT}}$

$\mathrm{Cl}_{\mathrm{OGTT}}=\mathrm{p} 4 \times\left(\left(\mathrm{p} 1 \times \mathrm{D}_{0}-\mathrm{V} \times(\mathrm{Glu} 120-\mathrm{Glu} 90) / 30\right) / \mathrm{Glu} 90+\mathrm{p} 3 / \mathrm{Glu} 0\right) /(\operatorname{Ins} 90-\operatorname{Ins} 0+\mathrm{p} 2)(22)$

*Glu0, Glu90, Glu120: glucose concentration values ( $\mathrm{mmol} / \mathrm{L})$, Ins0, Ins90: insulin concentration values ( $\mathrm{pmol} / \mathrm{L}), \mathrm{D}_{0}$ : glucose dose of the OGTT ( $\mathrm{mmol} / \mathrm{m}^{2}$, i.e. normalized for body surface area), V: glucose distribution volume $=10^{4} \mathrm{ml} / \mathrm{kg}$, Glu $\mathrm{CLAMP}_{\text {: }}$ clamp glucose concentration $=5 \mathrm{mmol} / \mathrm{L}, \mathrm{p} 1=6.50, \mathrm{p} 2=1951$, p3 $=4514, \mathrm{p} 4=792, \mathrm{p} 5=11.8 \times 10^{-3}, \mathrm{p} 6=173$.

PREDIM is calculated using the following equation:

$\log _{e}($ PREDIM $)=A+B \times \log _{e}(O G I S)+C \times \log _{e}(B M I)+D \times \log _{e}\left(\right.$ Glu120) $+E \times \log _{e}(\operatorname{Ins} 0)(21)$

*Glu120: glucose concentration values (mmol/L), Ins0: insulin concentration values (pmol/L), $A=$ 2.8846219, $B=0.5208520, C=-0.8223363, D=-0.4191242, E=-0.2427896$

The equivalence test in subgroups showed that the clamp-derived $M$ value and PREDIM value were similar for comparison of subgroups based on glucose tolerance (normal glucose tolerance, impaired fasting glucose, impaired glucose tolerance, type 2 diabetes) and BMI (lean, overweight, obesity) (21).

\section{Muscle strength measurement}

We evaluated the isokinetic muscle strength of the knee extensors using a dynamometer (BIODEX system 3 or 4: Biodex Medical Systems, Upton, NY, USA) (17). Participants were stabilized in the examination chair with shoulder and abdominal straps. The isokinetic peak torques of the knee extensors were measured at an angular velocity of 60 degrees per second. During the test, participants were encouraged to exert maximal muscle force. The isokinetic peak torques of the knee extensors were adjusted by body weight according to the following formula: isokinetic peak torques $(\mathrm{Nm}) /$ body weight $(\mathrm{kg})(17)$.

\section{Evaluation of silent lacunar infarcts}


The whole brain of each subject was scanned with a 0.3-T clinical MR scanner (AIRIS Vento, Hitachi, Tokyo, Japan). Sequences included axial three-dimensional (3D) time-of-flight magnetic resonance angiography (repetition time (TR), $35 \mathrm{~ms}$; echo time (TE), $7.1 \mathrm{~ms}$; and slice thickness, $1.2 \mathrm{~mm}$ ), T2*weighted gradient echo (T2*-WI) imaging (TR, 1000 ms; TE, 45 ms; flip angle, 20*; and slice thickness, 5 $\mathrm{mm}$ ) and fluid-attenuated inversion recovery (FLAIR) imaging (TR, 11,000 ms; TE, 100 ms; inversion time (TI), $2000 \mathrm{~ms}$; and slice thickness, $5 \mathrm{~mm}$ ). The evaluation of lacunar infarcts was conducted by an experienced neuroradiologist based on axial T2*-WI and FLAIR images. The radiologist was blinded to all clinical data. Lacunar infarcts were defined as infarcts of 3-15 $\mathrm{mm}$ in diameter located in the deep cerebral white matter, basal ganglia, or pons, and that were presumed to result from the occlusion of a single, small, perforating artery supplying the subcortical area of the brain $(23,24)$.

\section{Other measurements}

BMI was calculated as weight in kilograms divided by the square of height in meters $\left(\mathrm{kg} / \mathrm{m}^{2}\right)$. Physical activity was evaluated using the International Physical Activity Questionnaire (IPAQ), which assesses different types of physical activity, such as walking and moderate- and high-intensity activities $(25,26)$. Hypertension was defined as systolic blood pressure (SBP) $\geq 140 \mathrm{mmHg}$ and/or diastolic blood pressure $(D B P) \geq 90 \mathrm{mmHg}$ or current treatment with antihypertensive medications. Diabetes was defined as a combination of fasting plasma glucose $\geq 126 \mathrm{mg} / \mathrm{dL}$ and/or a 2-hour glucose level $\geq 200 \mathrm{mg} / \mathrm{dL}$ after the 75-g OGTT, and hemoglobin A1c $\geq 6.5 \%$, or currently treatment with medication for diabetes mellitus. Dyslipidemia was defined as low-density lipoprotein cholesterol (LDL-C) $\geq 140 \mathrm{mg} / \mathrm{dL}$ and/or highdensity lipoprotein cholesterol (HDL-C) $<40 \mathrm{mg} / \mathrm{dL}$ and/or triglycerides $(\mathrm{TG}) \geq 150 \mathrm{mg} / \mathrm{dL}$, or current treatment with lipid-lowering agents. Cardiovascular disease was defined by a medical history of ischemic heart disease or heart failure.

\section{Statistical analysis}

Male and female participants were separately divided into three groups (High, Medium, and Low) based on the standard deviation (SD) of sex-specific values of PREDIM and divided three groups (High, Medium, and Low) based on the tertiles of sex and age (65-69, 70-74, 75-79, and 80-84 years)-specific value of muscle strength (Table 1). Then, participants of both sexes were categorized into nine groups according to the combination of these values. Data are presented as mean \pm SD or number (\%). Characteristics of the three insulin sensitivity groups were analyzed for trend by the Jonckheere-Terpstra test (continuous variables) and Cochran-Armitage trend test (categorical variables). All statistical tests were two-sided with a 5\% significant level. Logistic regression analysis was used to estimate odds ratios (ORs) and 95\% confidence intervals (Cls) for the associations between the prevalence of silent lacunar infarcts and insulin sensitivity, muscle strength, and their combination, with adjustment for age (continuous variable), sex (male or female), and other potential risk factors. In this study, three models were applied for regression analysis: model 1 was adjusted for age and sex; model 2 was adjusted for muscle strength (continuous variable) or insulin sensitivity (continuous variable), physical activity level (continuous variable), and cigarette smoking (never, past and current smoker), and incorporated model 1; and model 3 
was adjusted for hypertension (yes or no), diabetes (yes or no), dyslipidemia (yes or no), and cardiovascular disease (yes or no), and incorporated model 2.

Table 1

Cut-off values for insulin sensitivity and muscle strength

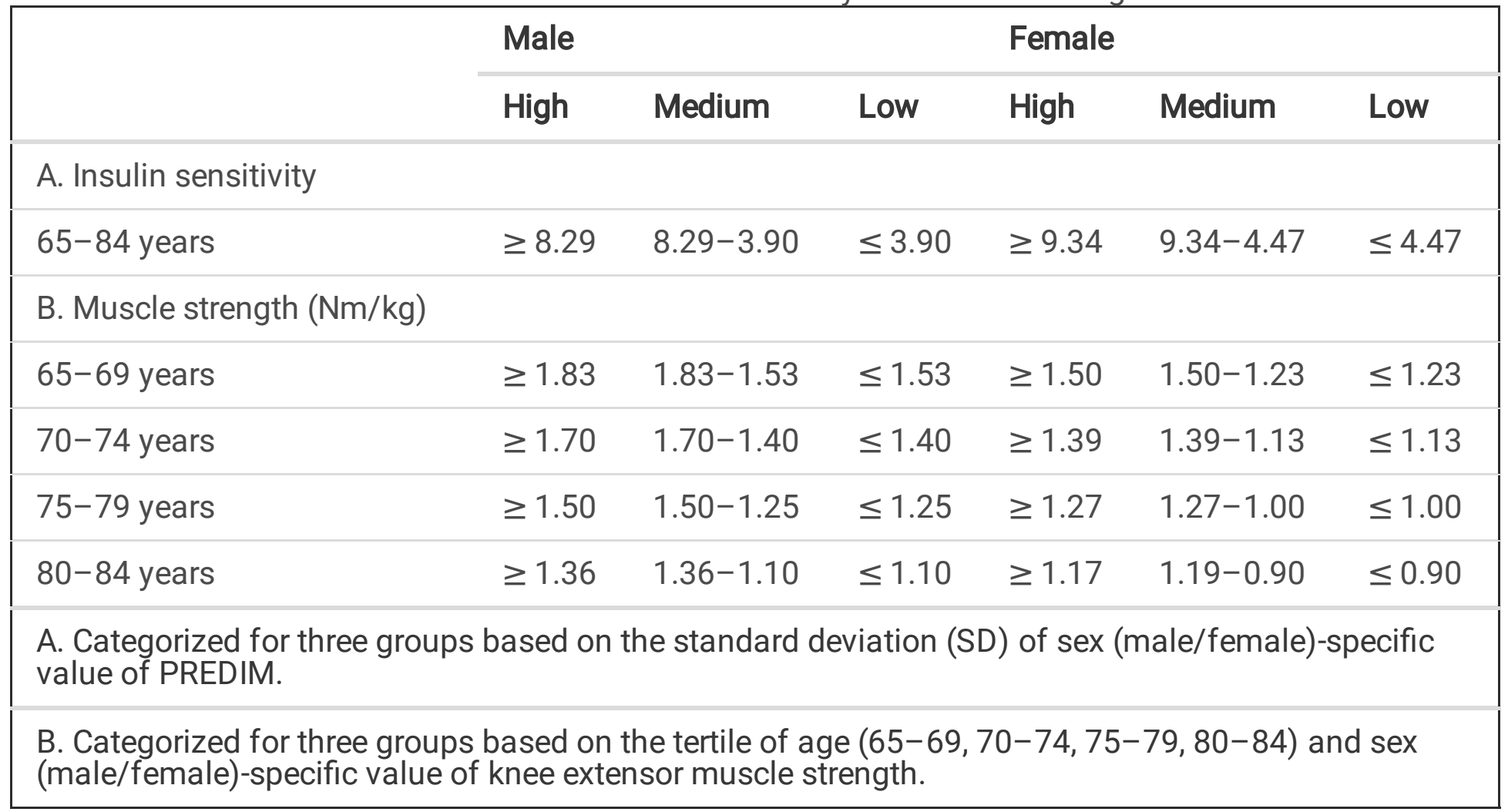

\section{Results}

\section{Characteristics according to insulin sensitivity groups}

Of the 1531 participants in this cohort, 251 (16.4\%) had silent lacunar infarcts. The mean age of participants was $73.0 \pm 5.4$ years. The mean insulin sensitivity value (PREDIM) was $6.09 \pm 2.18$ in men and $6.90 \pm 2.43$ in women. The cut-off value for low insulin sensitivity (under - 1SD) was similar to that in Japanese diabetic patients $(27,28)$. Characteristics of the subjects in the three groups divided according to insulin sensitivity are shown in Table 2 . The prevalence of silent lacunar infarcts was inversely associated with insulin sensitivity. Reduced insulin sensitivity was associated with higher age, higher BMI, lower physical activity, and lower muscle strength. Similarly, we observed associations between reduced insulin sensitivity and higher prevalences of hypertension, diabetes, dyslipidemia, and cardiovascular disease. 
Table 2

Characteristics according to insulin sensitivity categories

\begin{tabular}{|c|c|c|c|c|c|}
\hline & All & $\begin{array}{l}\text { High } \\
\text { (+ 1SD) }\end{array}$ & Medium & $\begin{array}{l}\text { Low } \\
\text { (-1SD) }\end{array}$ & $\begin{array}{l}p \text { for } \\
\text { trend* }\end{array}$ \\
\hline$N$ & 1531 & 236 & 1057 & 238 & \\
\hline$(M / F)$ & $(627 / 904)$ & $(96 / 140)$ & $(431 / 626)$ & $(100 / 138)$ & \\
\hline PREDIM & $6.6 \pm 2.4$ & $10.5 \pm 1.7$ & $6.4 \pm 1.3$ & $3.5 \pm 0.6$ & \\
\hline Silent lacunar infarcts & $\begin{array}{l}251 \\
(16.4 \%)\end{array}$ & $23(9.7 \%)$ & $\begin{array}{l}174 \\
(16.5 \%)\end{array}$ & $54(22.7 \%)$ & $<0.001$ \\
\hline Age, years & $73.0 \pm 5.4$ & $71.9 \pm 5.3$ & $73.0 \pm 5.3$ & $74.1 \pm 5.3$ & $<0.001$ \\
\hline Height, cm & $158.0 \pm 8.8$ & $158.1 \pm 8.3$ & $158.1 \pm 8.7$ & $157.3 \pm 9.4$ & 0.354 \\
\hline Body weight, kg & $56.9 \pm 10.2$ & $49.2 \pm 8.2$ & $57.1 \pm 9.3$ & $64.1 \pm 10.7$ & $<0.001$ \\
\hline Body mass index, $\mathrm{kg} / \mathrm{m}^{2}$ & $22.7 \pm 3.0$ & $19.6 \pm 2.1$ & $22.7 \pm 2.4$ & $25.8 \pm 3.1$ & $<0.001$ \\
\hline $\begin{array}{l}\text { Skeletal Muscle Index, } \mathrm{kg} / \\
\mathrm{m}^{2}\end{array}$ & $6.4 \pm 1.0$ & $6.1 \pm 0.9$ & $6.4 \pm 0.9$ & $6.7 \pm 1.0$ & $<0.001$ \\
\hline Percent body fat, \% & $28.3 \pm 7.3$ & $21.1 \pm 5.6$ & $28.4 \pm 6.2$ & $34.6 \pm 6.7$ & $<0.001$ \\
\hline Waist circumference, $\mathrm{cm}$ & $86.5 \pm 9.2$ & $78.2 \pm 7.2$ & $86.5 \pm 7.8$ & $94.7 \pm 9.5$ & $<0.001$ \\
\hline Hypertension & $\begin{array}{l}991 \\
(64.7 \%)\end{array}$ & $\begin{array}{l}109 \\
(46.2 \%)\end{array}$ & $\begin{array}{l}680 \\
(64.3 \%)\end{array}$ & $\begin{array}{l}202 \\
(84.9 \%)\end{array}$ & $<0.001$ \\
\hline Diabetes & $\begin{array}{l}186 \\
(12.1 \%)\end{array}$ & $2(0.8 \%)$ & $79(7.5 \%)$ & $\begin{array}{l}105 \\
(44.1 \%)\end{array}$ & $<0.001$ \\
\hline Hyperlipidemia & $\begin{array}{l}957 \\
(62.5 \%)\end{array}$ & $\begin{array}{l}100 \\
(42.4 \%)\end{array}$ & $\begin{array}{l}660 \\
(62.4 \%)\end{array}$ & $\begin{array}{l}197 \\
(82.8 \%)\end{array}$ & $<0.001$ \\
\hline Cardiovascular disease & $67(4.4 \%)$ & $1(0.4 \%)$ & $48(4.5 \%)$ & $18(7.6 \%)$ & $<0.001$ \\
\hline \multicolumn{6}{|l|}{ Smoking } \\
\hline Past smoking & $\begin{array}{l}500 \\
(32.7 \%)\end{array}$ & 77 (32.6\%) & $\begin{array}{l}338 \\
(32.0 \%)\end{array}$ & $85(35.7 \%)$ & 0.472 \\
\hline Current smoking & $114(7.4 \%)$ & $25(10.6 \%)$ & $80(7.6 \%)$ & $9(3.8 \%)$ & 0.005 \\
\hline Muscle strength, $\mathrm{Nm}$ & $76.5 \pm 27.3$ & $71.6 \pm 22.6$ & $77.5 \pm 27.4$ & $76.6 \pm 30.3$ & 0.203 \\
\hline
\end{tabular}

Data are mean \pm SD or number (\%)

*p for trend was analyzed by the Jonckheere Terpstra test (continuous variables) and CochranArmitage trend test (categorical variables). 


\begin{tabular}{|c|c|c|c|c|c|}
\hline & All & $\begin{array}{l}\text { High } \\
\text { (+ 1SD) }\end{array}$ & Medium & $\begin{array}{l}\text { Low } \\
\text { (-1SD) }\end{array}$ & $\begin{array}{l}p \text { for } \\
\text { trend* }\end{array}$ \\
\hline Muscle strength, $\mathrm{Nm} / \mathrm{kg}$ & $\begin{array}{l}133.7 \pm \\
37.4\end{array}$ & $\begin{array}{l}144.6 \pm \\
34.1\end{array}$ & $\begin{array}{l}134.8 \pm \\
37.2\end{array}$ & $\begin{array}{l}118.1 \pm \\
36.6\end{array}$ & $<0.001$ \\
\hline $\begin{array}{l}\text { Physical activity, } \\
\text { METs/hour/week }\end{array}$ & $44.1 \pm 48.0$ & $57.9 \pm 62.6$ & $42.5 \pm 45.8$ & $37.6 \pm 37.4$ & $<0.001$ \\
\hline Sedentary time, hour & $6.0 \pm 3.6$ & $5.8 \pm 3.5$ & $6.0 \pm 3.6$ & $6.4 \pm 3.6$ & 0.072 \\
\hline Dietary intake, kcal & $\begin{array}{l}1963.6 \pm \\
594.1\end{array}$ & $\begin{array}{l}1979.2 \pm \\
593.7\end{array}$ & $\begin{array}{l}1966.4 \pm \\
590.0\end{array}$ & $\begin{array}{l}1936.6 \pm \\
614.0\end{array}$ & 0.387 \\
\hline Protein intake, $g$ & $83.1 \pm 30.6$ & $81.4 \pm 29.9$ & $83.2 \pm 30.2$ & $84.3 \pm 33.1$ & 0.502 \\
\hline Fat intake, $g$ & $61.6 \pm 22.0$ & $59.5 \pm 21.0$ & $61.8 \pm 21.9$ & $63.0 \pm 23.0$ & 0.101 \\
\hline Carbohydrate intake, $g$ & $\begin{array}{l}242.5 \pm \\
82.5\end{array}$ & $\begin{array}{l}250.5 \pm \\
82.5\end{array}$ & $\begin{array}{l}242.3 \pm \\
82.0\end{array}$ & $\begin{array}{l}235.6 \pm \\
84.1\end{array}$ & 0.013 \\
\hline Salt, $g$ & $12.5 \pm 4.1$ & $12.2 \pm 3.9$ & $12.5 \pm 4.0$ & $12.8 \pm 4.4$ & 0.204 \\
\hline Alcohol, $g$ & $13.3 \pm 23.4$ & $14.5 \pm 24.7$ & $13.5 \pm 23.4$ & $10.9 \pm 21.8$ & 0.002 \\
\hline $\begin{array}{l}\text { Systolic blood pressure, } \\
\mathrm{mmHg}\end{array}$ & $\begin{array}{l}136.4 \pm \\
17.1\end{array}$ & $\begin{array}{l}131.3 \pm \\
18.6\end{array}$ & $\begin{array}{l}136.4 \pm \\
17.0\end{array}$ & $\begin{array}{l}141.5 \pm \\
14.2\end{array}$ & $<0.001$ \\
\hline $\begin{array}{l}\text { Diastolic blood pressure, } \\
\mathrm{mmHg}\end{array}$ & $84.3 \pm 9.7$ & $81.3 \pm 10.0$ & $84.5 \pm 9.7$ & $86.1 \pm 8.9$ & $<0.001$ \\
\hline $\begin{array}{l}\text { Fasting plasma insulin, } \\
\mu U / m L\end{array}$ & $4.8 \pm 3.2$ & $2.2 \pm 0.8$ & $4.5 \pm 2.0$ & $9.1 \pm 4.7$ & $<0.001$ \\
\hline $\begin{array}{l}\text { Fasting plasma glucose, } \\
\mathrm{mg} / \mathrm{dL}\end{array}$ & $\begin{array}{l}100.1 \pm \\
16.1\end{array}$ & $90.1 \pm 6.7$ & $97.9 \pm 11.2$ & $\begin{array}{l}120.0 \pm \\
23.7\end{array}$ & $<0.001$ \\
\hline$H b A 1 c, \%$ & $5.8 \pm 0.6$ & $5.5 \pm 0.3$ & $5.8 \pm 0.4$ & $6.4 \pm 0.8$ & $<0.001$ \\
\hline Total cholesterol, mg/dL & $\begin{array}{l}207.3 \pm \\
36.0\end{array}$ & $\begin{array}{l}212.2 \pm \\
32.7\end{array}$ & $\begin{array}{l}208.1 \pm \\
36.3\end{array}$ & $\begin{array}{l}199.0 \pm \\
36.7\end{array}$ & $<0.001$ \\
\hline LDL cholesterol, mg/dL & $\begin{array}{l}122.1 \pm \\
30.6\end{array}$ & $\begin{array}{l}123.1 \pm \\
28.9\end{array}$ & $\begin{array}{l}122.8 \pm \\
30.8\end{array}$ & $\begin{array}{l}118.1 \pm \\
31.2\end{array}$ & 0.057 \\
\hline HDL cholesterol, mg/dL & $64.7 \pm 16.6$ & $72.4 \pm 16.2$ & $64.8 \pm 16.2$ & $56.6 \pm 14.9$ & $<0.001$ \\
\hline Triglycerides, $m g / d L$ & $98.5 \pm 54.8$ & $73.7 \pm 33.5$ & $97.0 \pm 51.9$ & $\begin{array}{l}129.9 \pm \\
68.4\end{array}$ & $<0.001$ \\
\hline $\begin{array}{l}\text { Aspartate } \\
\text { aminotransferase, IU/L }\end{array}$ & $23.4 \pm 9.5$ & $23.6 \pm 5.8$ & $22.6 \pm 8.5$ & $26.9 \pm 14.5$ & 0.705 \\
\hline \multicolumn{6}{|c|}{ Data are mean \pm SD or number (\%) } \\
\hline
\end{tabular}




\begin{tabular}{|c|c|c|c|c|c|}
\hline & All & $\begin{array}{l}\text { High } \\
\text { (+1SD) }\end{array}$ & Medium & $\begin{array}{l}\text { Low } \\
\text { (-1SD) }\end{array}$ & $\begin{array}{l}p \text { for } \\
\text { trend* }\end{array}$ \\
\hline $\begin{array}{l}\text { Alanine aminotransferase, } \\
I U / L\end{array}$ & $19.2 \pm 11.1$ & $16.3 \pm 5.8$ & $18.2 \pm 8.8$ & $26.5 \pm 18.6$ & $<0.001$ \\
\hline y-glutamyl transferase, IU/L & $30.7 \pm 36.5$ & $23.7 \pm 22.5$ & $30.0 \pm 37.7$ & $40.5 \pm 39.9$ & $<0.001$ \\
\hline Serum albumin, $g / d L$ & $43.0 \pm 3.7$ & $42.6 \pm 4.3$ & $43.0 \pm 3.6$ & $43.4 \pm 3.8$ & 0.033 \\
\hline Creatinine, $\mathrm{mg} / \mathrm{dL}$ & $0.8 \pm 0.3$ & $0.8 \pm 0.3$ & $0.8 \pm 0.4$ & $0.8 \pm 0.2$ & 0.515 \\
\hline Total adiponectin, $\mu \mathrm{g} / \mathrm{ml}$ & $12.4 \pm 6.6$ & $16.6 \pm 7.8$ & $12.2 \pm 6.0$ & $9.4 \pm 5.6$ & $<0.001$ \\
\hline 25-hydroxyvitamin $\mathrm{D}, \mathrm{ng} / \mathrm{mL}$ & $19.1 \pm 5.1$ & $19.3 \pm 5.7$ & $19.2 \pm 5.1$ & $18.6 \pm 4.7$ & 0.197 \\
\hline \multicolumn{6}{|c|}{ Data are mean \pm SD or number (\%) } \\
\hline
\end{tabular}

\section{Insulin sensitivity, muscle strength, and silent lacunar infarcts}

Insulin sensitivity was weakly but significantly correlated with muscle strength (Fig. 1, r $=0.184, p<$ 0.001). Table 3 shows the OR for silent lacunar infarcts in each group. The age- and sex-adjusted model revealed that both reduced insulin sensitivity and reduced muscle strength were significantly associated with a higher OR for silent lacunar infarcts (insulin sensitivity: High, 1.00 [reference]; Medium, 1.67 [95\%Cl: 1.05-2.67]; Low: 2.24 [95\% Cl: 1.31-3.84], p for trend 0.003; muscle strength: High, 1.00 [reference]; Medium, 1.48 [95\%Cl: 1.03-2.11]; Low: 1.65 [95\% Cl: 1.16-2.35], p for trend 0.006). Even after adjustment for risk factors such as muscle strength (model 3), the lowest insulin sensitivity group (under - 1SD) was associated with a higher OR for silent lacunar infarcts (High: 1.00 [reference]; Medium, 1.53 [95\%Cl: 0.94-2.48]; Low, 1.86 [95\% Cl: 1.02-3.39]). Furthermore, the linear trend across the three groups was significant ( $p$ for trend: 0.047 ). Additionally, the lowest muscle strength group was associated with a higher OR for silent lacunar infarcts even with adjustment for insulin sensitivity and other parameters in model 3 (High, 1.00 [reference]; Medium, 1.40 [95\%Cl: 0.98-2.02]; Low: 1.49 [95\%Cl: 1.04-2.15], p for trend 0.037). These data demonstrate that impaired insulin sensitivity and reduced muscle strength are each independently associated with higher risk for silent lacunar infarcts. 
Table 3

Associations between insulin sensitivity and silent lacunar infarcts

\section{Odds $(95 \% \mathrm{Cl})$}

Insulin sensitivity

High ( $\geq 1 S D)$

Medium

Low $(\leq-1 S D)$

p for trend

Muscle strength

High

Medium

Low

p for trend

Age (per 1 year)

Sex

Male

Female

Smoking

Never

Past

Current

Physical activity

(per METs/hour/week)

Hypertension (yes)
Model 1

Model 2

1.00

$1.64(1.02-2.63)$

$2.06(1.19-3.58)$

0.011

0.003

1.00

1.00

$1.39(0.97-2.00)$

$1.53(1.06-2.19)$

0.025

0.006

$1.11(1.09-1.14)$

$1.10(1.07-1.13)$

$1.09(1.06-1.13)$

1.48(1.03-2.11)

$1.65(1.16-2.35)$

1.00

1.00

$0.79(0.54-1.16)$

$0.88(0.66-1.17)$

1.00

$0.89(0.61-1.29)$
Model 3

1.00

$1.53(0.94-2.48)$

$1.86(1.02-3.39)$

0.047 


\section{Odds $(95 \% \mathrm{Cl})$}

Diabetes (yes)

$0.92(0.59-1.44)$

Hyperlipidemia (yes)

$0.87(0.64-1.18)$

Cardiovascular disease (yes)

$1.87(1.06-3.30)$

Model 1 was adjusted for age and sex.

Model 2 was adjusted for muscle strength or insulin sensitivity, smoking, physical activity, and incorporated model 1.

Model 3 was adjusted for hypertension, diabetes, dyslipidemia, cardiovascular disease, and incorporated model 2.

\section{Combination of muscle strength and muscle insulin sensitivity}

Next, to investigate the combined effect of reduced muscle strength and insulin sensitivity on the risk for silent lacunar infarcts, the participants were categorized into nine groups according to combinations of insulin sensitivity and muscle strength, and the OR for silent lacunar infarcts in each group is shown in Fig. 2. After adjusting for age, hypertension, and other potential risk factors, OR for silent lacunar infarcts was significantly higher in 4 groups with either insulin sensitivity or muscle strength was not "High" compared with the group with High-insulin sensitivity and High-muscle strength. Especially, subjects with the Low-insulin sensitivity and Low-muscle strength were 4.33 times ( $95 \% \mathrm{Cl}$ : $1.64-11.45)$ more likely to have silent lacunar infarcts than those with the High-insulin sensitivity and High-muscle strength. These results suggest that insulin resistance and reduced muscle strength synergistically increase the risk for silent lacunar infarcts.

\section{Discussion}

In this study, we investigated whether reduced insulin sensitivity and muscle strength were independent risk factors for silent lacunar infarcts in 1531 elderly subjects living in an urban area in Tokyo, Japan. We also evaluated the combined effect of insulin sensitivity and muscle strength on silent lacunar infarcts. In this cohort, 251 (16.4\%) subjects had silent lacunar infarcts. After full adjustment for potential risk factors, we found that impaired insulin sensitivity and reduced muscle strength were independently associated with an increased risk for silent lacunar infarcts. Furthermore, we found that subjects with the lowest insulin sensitivity and muscle strength had a 4.33-fold (95\%Cl: 1.64-11.45) higher risk for silent lacunar infarcts than those in the groups with the highest values for both parameters.

This study showed that lower insulin sensitivity evaluated by PREDIM was independently associated with higher risk of silent lacunar infarcts after full adjustment for potential risk factors. Most previous epidemiological studies used HOMA-IR as an index of insulin sensitivity; however, the accuracy of the index remains questionable. For example, Kang et al. suggested that the correlation between HOMA-IR 
and the glucose disposal rate measured by hyperinsulinemic euglycemic clamp is lower in subjects with lower BMI, lower beta cell function, and higher fasting glucose levels, such as lean individuals with type 2 diabetes mellitus with insulin secretory defects (29). Thus, several studies excluded patients with diabetes mellitus due to inaccurate estimation of insulin resistance by HOMA-IR (30-32). Of note, one such study demonstrated that insulin resistance evaluated by HOMA-IR was independently associated with silent lacunar infarcts (14). Based on this background, we used PREDIM as the index of insulin sensitivity since its accuracy has been validated in subjects with broad ranges of $\mathrm{BMI}$, age, and glycemic control, including diabetic patients, and we successfully demonstrated the association between insulin resistance and silent lacunar infarcts and included individuals with diabetes mellitus.

This study also showed that impaired insulin sensitivity and reduced muscle strength were independently associated with increased risk for silent lacunar infarcts, and the two factors demonstrated a significant combined effect. PREDIM is correlated with the glucose infusion rate during hyperinsulinemic euglycemic clamp (21), mainly reflecting insulin sensitivity in muscle (13). Thus, the present study suggested that two muscle characteristics, strength and insulin sensitivity, are independently and synergistically associated with silent lacunar infarcts. From a biological viewpoint, muscle strength and muscle insulin sensitivity are regulated differently. It has been reported that reduced muscle strength is caused by several defects, such as neurological dysfunction (33), impaired Ca handling in myocytes (34) and reduced muscle mass (35). On the other hand, muscle insulin resistance has been reported to be induced by different defects, such as impaired insulin signal transduction due to low-grade inflammation and intramyocellular lipid accumulation (36). In fact, similar to previous studies $(18,19)$, the present study revealed only a weak overall association between muscle strength and insulin sensitivity, and this association varied widely between individuals (Fig. 1), suggesting a different etiology of reduced muscle strength and insulin resistance.

The underlying mechanisms linking impaired insulin sensitivity, reduced muscle strength, and silent lacunar infarcts are not yet fully understood. Atherosclerosis has been recognized as a cause of silent lacunar infarcts (24), and it has been suggested that insulin resistance induces atherosclerosis (37). Thus, lower insulin sensitivity might cause silent lacunar infarcts via atherosclerosis. In terms of muscle strength, previous prospective studies showed that reduced muscle strength at 18 years of age was associated with an increased risk of ischemic stroke later in life $(15,38)$. Thus, by an undefined mechanism, lower muscle strength is associated with silent lacunar infarcts. Alternatively, it is also possible that silent lacunar infarcts cause lower muscle strength. In fact, patients with silent lacunar infarcts were shown to have diminished physical functions, for instance slow gait speed, loss of balance, and low physical activity (39-41).

The current study has several limitations. This cohort included only participants living in an urban part of Japan, and relatively healthy participants may have been included. However, the prevalence rate of silent lacunar infarcts (16.4\%) in the present study was similar to that in a previous study in Japanese (1), and thus our data should be reflective of elderly individuals living in an urban area of Japan. In addition, this study was cross-sectional in nature, and thus causal relationships have not yet been determined. Further 
prospective and interventional studies are required to clarify the roles of muscle insulin sensitivity and strength on the incidence of silent lacunar infarcts.

\section{Conclusions}

Impaired insulin sensitivity and reduced muscle strength were independently associated with a higher risk for silent lacunar infarcts, and the combination of both synergistically elevated this risk in Japanese elderly individuals.

\section{Declarations}

\section{Ethics approval and consent to participate:}

This study protocol was approved by the ethics committee of Juntendo University in November 2015 (Nos. 2015078, 2016138, 2016131, 2017121, and 2019085). This study is carried out in accordance with the principles outlined in the Declaration of Helsinki. All participants gave written informed consent at the orientation meeting. Participants were told that they had the right to withdraw from the trial at any time.

\section{Consent for publication:}

Not applicable

\section{Availability of data and materials:}

The datasets used and/or analyzed during the current study are available from the corresponding author on reasonable request.

\section{Competing interests:}

The authors declare that they have no competing interests.

\section{Funding:}

This work was supported by the Strategic Research Foundation at Private Universities (S1411006) and a KAKENHI (18H03184) grants from the Ministry of Education, Culture, Sports, Science, and Technology of Japan; the Mizuno Sports Promotion Foundation; and Mitsui Life Social Welfare Foundation.

\section{Authors' contributions:}

Y.S., H.K., and Y.T. performed the research and contributed to the study design, data collection, interpretation of results, and writing and editing of the manuscript. Y.S., H.K., D.S., S.K., and R.S. participated in data collection and analysis, and contributed to the discussion. N.H., S.A., Y.M., K.S., H.D., M.I., and K.K. reviewed and revised the manuscript. S.N. provided advice regarding the statistical analysis. 
R.K. contributed to the discussion. H.W. contributed to the study design and reviewed and edited the manuscript.

\section{Acknowledgements:}

The authors would like to thank Dr. Mori H. for contributing to brain MRI evaluation, and Liu L., Aoki T., Nakagata T., Sato M., Yamazaki N., Hui H., and all staff at the Sportology Center for contributing to data collection.

\section{References}

1. Fanning JP, Wong AA, Fraser JF. The epidemiology of silent brain infarction: a systematic review of population-based cohorts. BMC Med. 2014;12:119.

2. Nakagawa T, Sekizawa K, Nakajoh K, Tanji H, Arai H, Sasaki H. Silent cerebral infarction: a potential risk for pneumonia in the elderly. J Intern Med. 2000;247(2):255-9.

3. Saji N, Kimura K, Shimizu H, Kita Y. Silent brain infarct is independently associated with arterial stiffness indicated by cardio-ankle vascular index (CAVI). Hypertens Res. 2012;35(7):756-60.

4. Fukuda K, Takashima Y, Hashimoto M, Uchino A, Yuzuriha T, Yao H. Early menopause and the risk of silent brain infarction in community-dwelling elderly subjects: the Sefuri brain MRI study. J Stroke Cerebrovasc Dis. 2014;23(5):817-22.

5. Bernick C, Kuller L, Dulberg C, Longstreth WT, Jr., Manolio T, Beauchamp N, et al. Silent MRI infarcts and the risk of future stroke: the cardiovascular health study. Neurology. 2001;57(7):1222-9.

6. Kobayashi S, Okada K, Koide H, Bokura H, Yamaguchi S. Subcortical silent brain infarction as a risk factor for clinical stroke. Stroke. 1997;28(10):1932-9.

7. Vermeer SE, Prins ND, den Heijer T, Hofman A, Koudstaal PJ, Breteler MM. Silent brain infarcts and the risk of dementia and cognitive decline. The New England journal of medicine. 2003;348(13):1215-22.

8. Newman AB, Gottdiener JS, McBurnie MA, Hirsch CH, Kop WJ, Tracy R, et al. Associations of subclinical cardiovascular disease with frailty. The journals of gerontology Series A, Biological sciences and medical sciences. 2001;56(3):M158-66.

9. Schernthaner G, Schernthaner-Reiter MH. Diabetes in the older patient: heterogeneity requires individualisation of therapeutic strategies. Diabetologia. 2018;61(7):1503-16.

10. Vermeer SE, Longstreth WT, Jr., Koudstaal PJ. Silent brain infarcts: a systematic review. Lancet Neurol. 2007;6(7):611-9.

11. Bokura H, Yamaguchi S, lijima K, Nagai A, Oguro H. Metabolic syndrome is associated with silent ischemic brain lesions. Stroke. 2008;39(5):1607-9.

12. Eckel RH, Grundy SM, Zimmet PZ. The metabolic syndrome. Lancet. 2005;365(9468):1415-28.

13. Takeno K, Tamura Y, Kawaguchi M, Kakehi S, Watanabe T, Funayama T, et al. Relation Between Insulin Sensitivity and Metabolic Abnormalities in Japanese Men With BMI of $23-25 \mathrm{~kg} / \mathrm{m}(2)$. The 
Journal of clinical endocrinology and metabolism. 2016;101(10):3676-84.

14. Lee JE, Shin DW, Yun JM, Kim SH, Nam YS, Cho B, et al. Insulin Resistance Is a Risk Factor for Silent Lacunar Infarction. Stroke. 2016;47(12):2938-44.

15. Aberg ND, Kuhn HG, Nyberg J, Waern M, Friberg P, Svensson J, et al. Influence of Cardiovascular Fitness and Muscle Strength in Early Adulthood on Long-Term Risk of Stroke in Swedish Men. Stroke. 2015;46(7):1769-76.

16. Sasaki H, Kasagi F, Yamada M, Fujita S. Grip strength predicts cause-specific mortality in middleaged and elderly persons. Am J Med. 2007;120(4):337-42.

17. Someya Y, Tamura Y, Kaga H, Sugimoto D, Kadowaki S, Suzuki R, et al. Reduced muscle strength of knee extensors is a risk factor for silent lacunar infarcts among Japanese elderly people: the Bunkyo Health Study. JCSM Clinical Reports. 2020.

18. Barzilay JI, Cotsonis GA, Walston J, Schwartz AV, Satterfield S, Miljkovic I, et al. Insulin resistance is associated with decreased quadriceps muscle strength in nondiabetic adults aged $>0 \mathrm{r}=70$ years. Diabetes care. 2009;32(4):736-8.

19. Abbatecola AM, Ferrucci L, Ceda G, Russo CR, Lauretani F, Bandinelli S, et al. Insulin resistance and muscle strength in older persons. The journals of gerontology Series A, Biological sciences and medical sciences. 2005;60(10):1278-82.

20. Someya Y, Tamura Y, Kaga H, Nojiri S, Shimada K, Daida H, et al. Skeletal muscle function and need for long-term care of urban elderly people in Japan (the Bunkyo Health Study): a prospective cohort study. BMJ Open. 2019;9(9):e031584.

21. Tura A, Chemello G, Szendroedi J, Gobl C, Faerch K, Vrbikova J, et al. Prediction of clamp-derived insulin sensitivity from the oral glucose insulin sensitivity index. Diabetologia. 2018;61(5):1135-41.

22. Mari A, Pacini G, Murphy E, Ludvik B, Nolan JJ. A model-based method for assessing insulin sensitivity from the oral glucose tolerance test. Diabetes care. 2001;24(3):539-48.

23. Smith EE, Saposnik G, Biessels GJ, Doubal FN, Fornage M, Gorelick PB, et al. Prevention of Stroke in Patients With Silent Cerebrovascular Disease: A Scientific Statement for Healthcare Professionals From the American Heart Association/American Stroke Association. Stroke. 2017;48(2):e44-e71.

24. Wardlaw JM, Smith C, Dichgans M. Mechanisms of sporadic cerebral small vessel disease: insights from neuroimaging. Lancet Neurol. 2013;12(5):483-97.

25. Craig CL, Marshall AL, Sjostrom M, Bauman AE, Booth ML, Ainsworth BE, et al. International physical activity questionnaire: 12-country reliability and validity. Medicine and science in sports and exercise. 2003;35(8):1381-95.

26. Murase N, Katsumura T, Ueda U, Inoue S, Shimomitsu T. International standardization of physical activity level: reliability and validity study of the Japanese version of the international physical activity questionnaire (IPAQ). (Kosei no Shihyo) J Health Welfare Stat. 2003;49:1-9.

27. Shigiyama F, Kumashiro N, Furukawa Y, Funayama T, Takeno K, Wakui N, et al. Characteristics of hepatic insulin-sensitive nonalcoholic fatty liver disease. Hepatol Commun. 2017;1(7):634-47. 
28. Furukawa Y, Tamura Y, Takeno K, Funayama T, Kaga H, Suzuki R, et al. Impaired peripheral insulin sensitivity in non-obese Japanese patients with type 2 diabetes mellitus and fatty liver. Journal of diabetes investigation. 2017;9(3):529-35.

29. Kang ES, Yun YS, Park SW, Kim HJ, Ahn CW, Song YD, et al. Limitation of the validity of the homeostasis model assessment as an index of insulin resistance in Korea. Metabolism: clinical and experimental. 2005;54(2):206-11.

30. Rundek T, Gardener H, Xu Q, Goldberg RB, Wright CB, Boden-Albala B, et al. Insulin resistance and risk of ischemic stroke among nondiabetic individuals from the northern Manhattan study. Arch Neurol. 2010;67(10):1195-200.

31. Nakamura K, Sakurai M, Miura K, Morikawa Y, Ishizaki M, Yoshita K, et al. Homeostasis model assessment of insulin resistance and the risk of cardiovascular events in middle-aged non-diabetic Japanese men. Diabetologia. 2010;53(9):1894-902.

32. Howard G, Wagenknecht LE, Kernan WN, Cushman M, Thacker EL, Judd SE, et al. Racial differences in the association of insulin resistance with stroke risk: the REasons for Geographic And Racial Differences in Stroke (REGARDS) study. Stroke. 2014;45(8):2257-62.

33. McKinnon NB, Connelly DM, Rice CL, Hunter SW, Doherty TJ. Neuromuscular contributions to the agerelated reduction in muscle power: Mechanisms and potential role of high velocity power training. Ageing Res Rev. 2017;35:147-54.

34. Eshima H, Tamura Y, Kakehi S, Nakamura K, Kurebayashi N, Murayama T, et al. Dysfunction of muscle contraction with impaired intracellular $\mathrm{Ca}(2+)$ handling in skeletal muscle and the effect of exercise training in male db/db mice. Journal of applied physiology. 2019;126(1):170-82.

35. Tieland M, Trouwborst I, Clark BC. Skeletal muscle performance and ageing. J Cachexia Sarcopenia Muscle. 2018;9(1):3-19.

36. Tamura Y, Tanaka Y, Sato F, Choi JB, Watada H, Niwa M, et al. Effects of diet and exercise on muscle and liver intracellular lipid contents and insulin sensitivity in type 2 diabetic patients. The Journal of clinical endocrinology and metabolism. 2005;90(6):3191-6.

37. Nigro J, Osman N, Dart AM, Little PJ. Insulin resistance and atherosclerosis. Endocr Rev. 2006;27(3):242-59.

38. Silventoinen K, Magnusson PK, Tynelius P, Batty GD, Rasmussen F. Association of body size and muscle strength with incidence of coronary heart disease and cerebrovascular diseases: a population-based cohort study of one million Swedish men. Int J Epidemiol. 2009;38(1):110-8.

39. Longstreth WT, Jr., Bernick C, Manolio TA, Bryan N, Jungreis CA, Price TR. Lacunar infarcts defined by magnetic resonance imaging of 3660 elderly people: the Cardiovascular Health Study. Arch Neurol. 1998;55(9):1217-25.

40. Rosano C, Brach J, Longstreth WT, Jr., Newman AB. Quantitative measures of gait characteristics indicate prevalence of underlying subclinical structural brain abnormalities in high-functioning older adults. Neuroepidemiology. 2006;26(1):52-60. 
41. Rosano C, Kuller LH, Chung H, Arnold AM, Longstreth WT, Jr., Newman AB. Subclinical brain magnetic resonance imaging abnormalities predict physical functional decline in high-functioning older adults. Journal of the American Geriatrics Society. 2005;53(4):649-54.

\section{Figures}

\section{Figure 1}

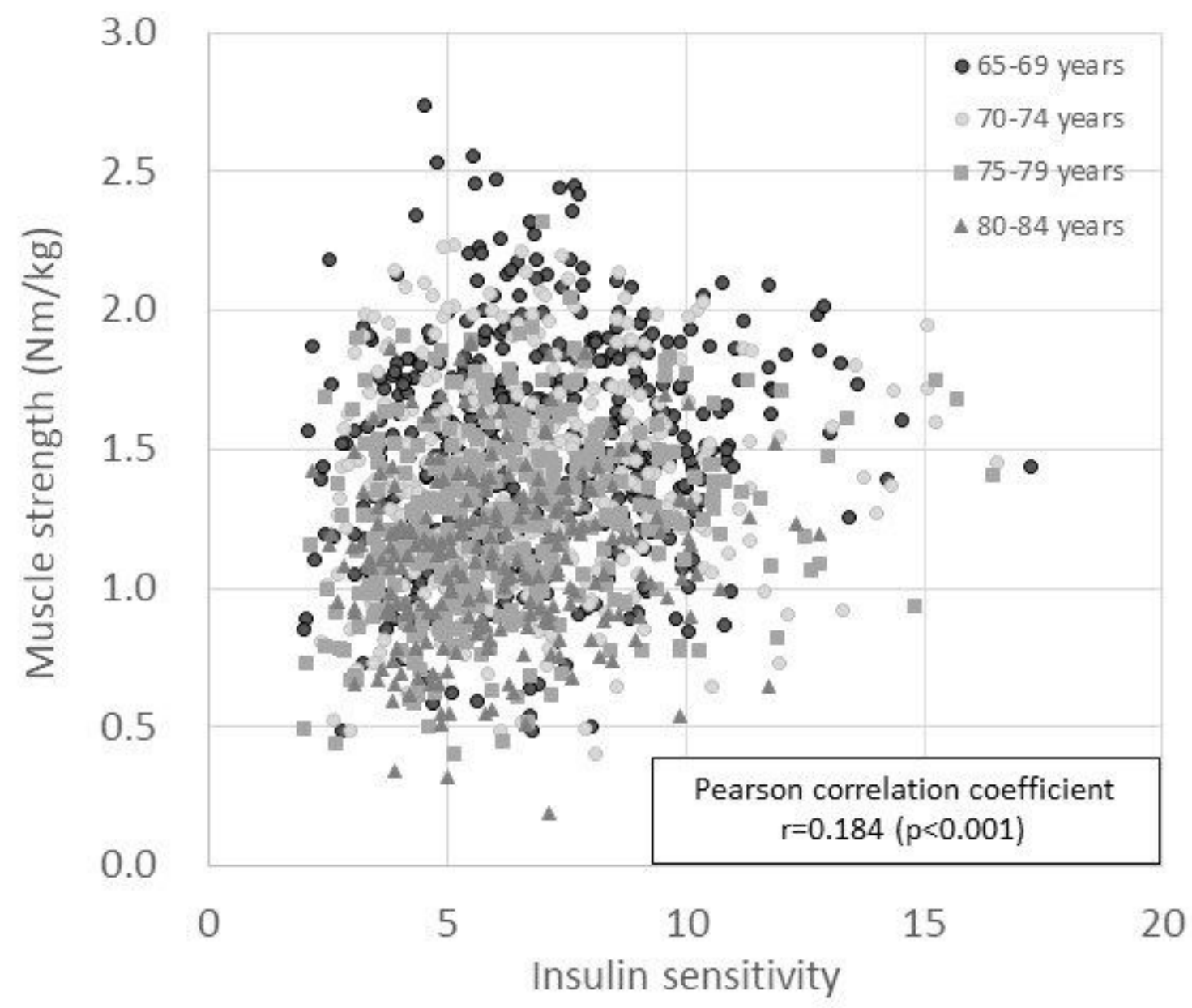

Figure 1

Combination of muscle strength and muscle insulin sensitivity 


\section{Figure 2}

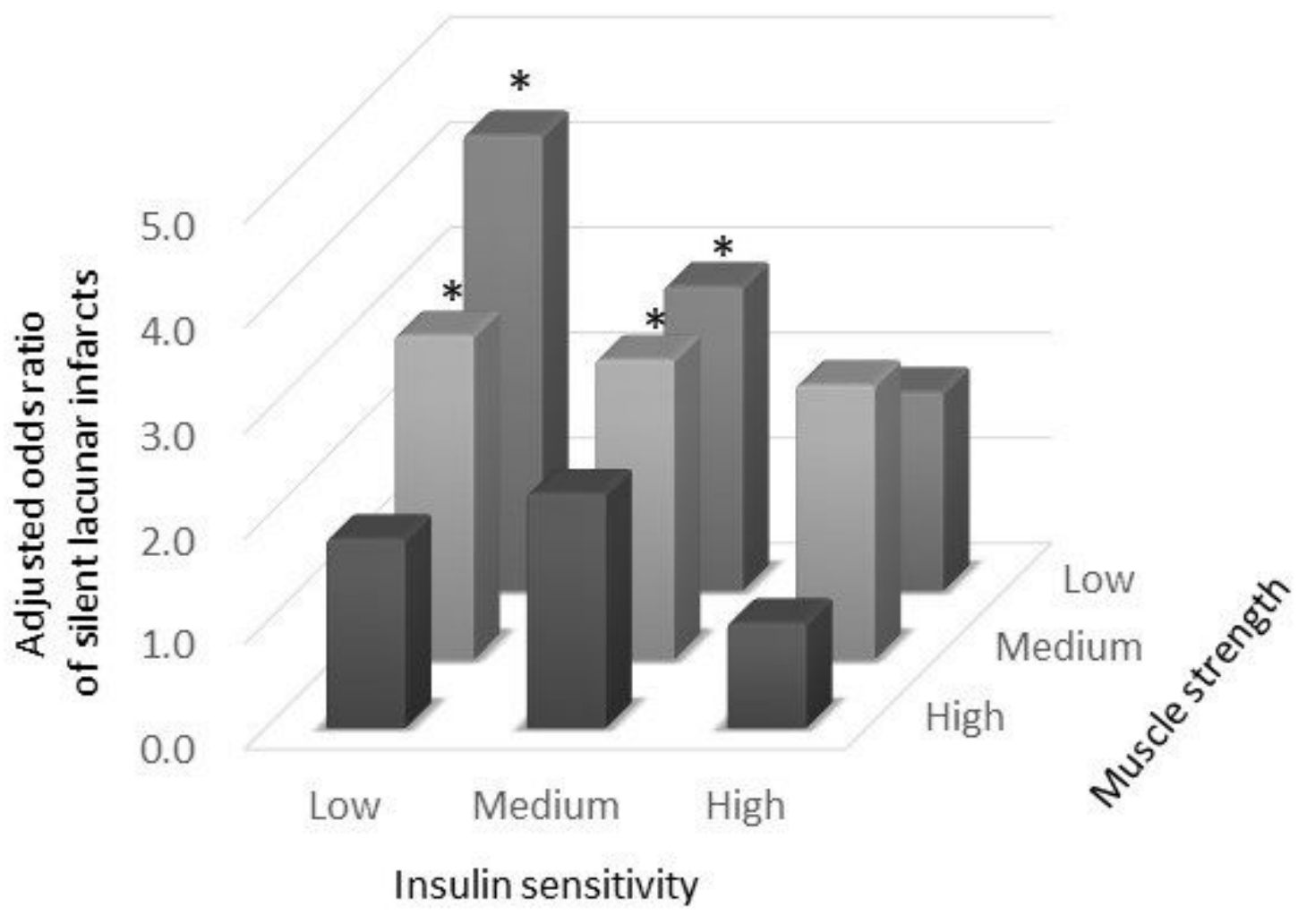

Figure 2

Insulin sensitivity, muscle strength, and silent lacunar infarcts 